Operative Techniken

Oper Orthop Traumatol 2019 $\cdot 31: 311-320$ https://doi.org/10.1007/s00064-019-0616-6 Eingegangen: 28. Oktober 2018

Überarbeitet: 25. Januar 2019

Angenommen: 2. April 2019

Online publiziert: 5. Juli 2019

() Der/die Autor(en) 2019

\section{Redaktion}

M. Mayer, München

Zeichner

H.J. Schütze, Köln

\section{Vorbemerkungen}

Die Inzidenz fokaler Kyphosen an der Brustwirbelsäule (BWS) ist gering. Die Fehlstellung hat neben Auswirkungen auf das Erscheinungsbild der Patienten auch gravierende medizinische Folgen. Im Vordergrund stehen zunehmende Schmerzen, schnelle Erschöpfbarkeit, Dyspnoe und neurologische Defizite [1]. Ursächlich waren in der Vergangenheit hauptsächlich ossäre Destruktionen von thorakalen Wirbeln bei Tuberkulose oder kongenitalen Fehlbildungen [2]. Mit Zunahme der Inzidenz Osteoporose-assoziierter Frakturen an der BWS wird es zukünftig zu einer Häufung von thorakalen kyphotischen Fehlstellungen kommen. Neben der Minderung der Knochenqualität erhöht insbesondere die Zunahme der Kyphose das Risiko für Anschlussfrakturen durch eine Verlagerung der Tragachse nach ventral. Therapieziele sind die Beseitigung der Fehlstellung in sagittaler und koronarer Ebene, Vorbeugung einer Rekyphosierung und eine Beschwerdebesserung bei den Patienten. Die technisch aufwändige „posterior vertebral column resection“ (PVCR) ist die operative Technik, die im Vergleich $\mathrm{zu}$ anderen Techniken (z.B. Smith-Peterson-Osteotomie oder Pedikelsubtraktionsosteotomie) die größte Deformitätenkorrektur an der BWS ermöglicht [3]. Die Operationstechnik ist

S. Sehmisch ${ }^{1}$ - W. Lehmann ${ }^{1} \cdot$ M. Dreimann ${ }^{2} \cdot$ S. Oberthür ${ }^{1} \cdot$ L. Viezens ${ }^{1} \cdot$ L. Weiser $^{1}$ 'Klinik für Unfallchirurgie, Orthopädie und Plastische Chirurgie, Universitätsmedizin Göttingen, Göttingen, Deutschland

${ }^{2}$ Klinik und Poliklinik für für Unfall-, Hand- und Wiederherstellungschirurgie, Spine Center, Universitätsklinikum Hamburg Eppendorf, Hamburg, Deutschland

\title{
Posterior vertebral column resection (PVCR) zur Korrektur von Hyperkyphosen bei osteoporotischen Frakturen der Brustwirbelsäule
}

extrem anspruchsvoll und sollte daher sehr kritisch indiziert werden.

\section{Operationsprinzip und -ziel}

Das Operationsprinzip ist in - Abb. 1 schematisch dargestellt. Ziel der Operation sind die Wiederherstellung des sagittalen Profils bei Hyperkyphose der thorakalen Wirbelsäule sowie die Dekompression des Spinalkanals mit gleichzeitiger dorsoventraler Spondylodese über einen isoliert dorsalen Zugang.

\section{Vorteile}

- Isoliert dorsaler Zugang zur Brustwirbelsäule

- Sehr gute Möglichkeit zur Deformitätenkorrektur

- Gute Darstellung der neuronalen Strukturen

- Vermeidung eines ventralen Zugangs mit Kompromittieren der Thoraxorgane

\section{Nachteile}

- Technisch sehr anspruchsvolle Operation

- Hoher Blutverlust möglich

- Intraoperative Instabilität der Brustwirbelsäule
- Gefahr der neurologischen Kompromittierung

\section{Indikationen}

- Hochgradige symptomatische thorakale Deformität mit Kyphose $>45^{\circ}$ (Cobb)

- Sekundäre Spinalkanalstenose bei hochgradiger thorakaler Kyphose

- Knöcherne Defekte bei Spondylodiszitis oder Tumoren

\section{Kontraindikationen}

- Allgemeine Kontraindikationen gegen Operationen

- ASA >3 (American Society of Anesthesiologists)

\section{Patientenaufklärung}

- Auftreten von neurologischen Komplikationen

- Hypästhesie im Bereich der Rhizotomie (selten bei monosegmentaler VCR)

- Hoher Blutverlust mit Gabe von Erythrozytenkonzentraten

- Postoperative Wundheilungsstörung mit Notwendigkeit von Folgeoperationen 

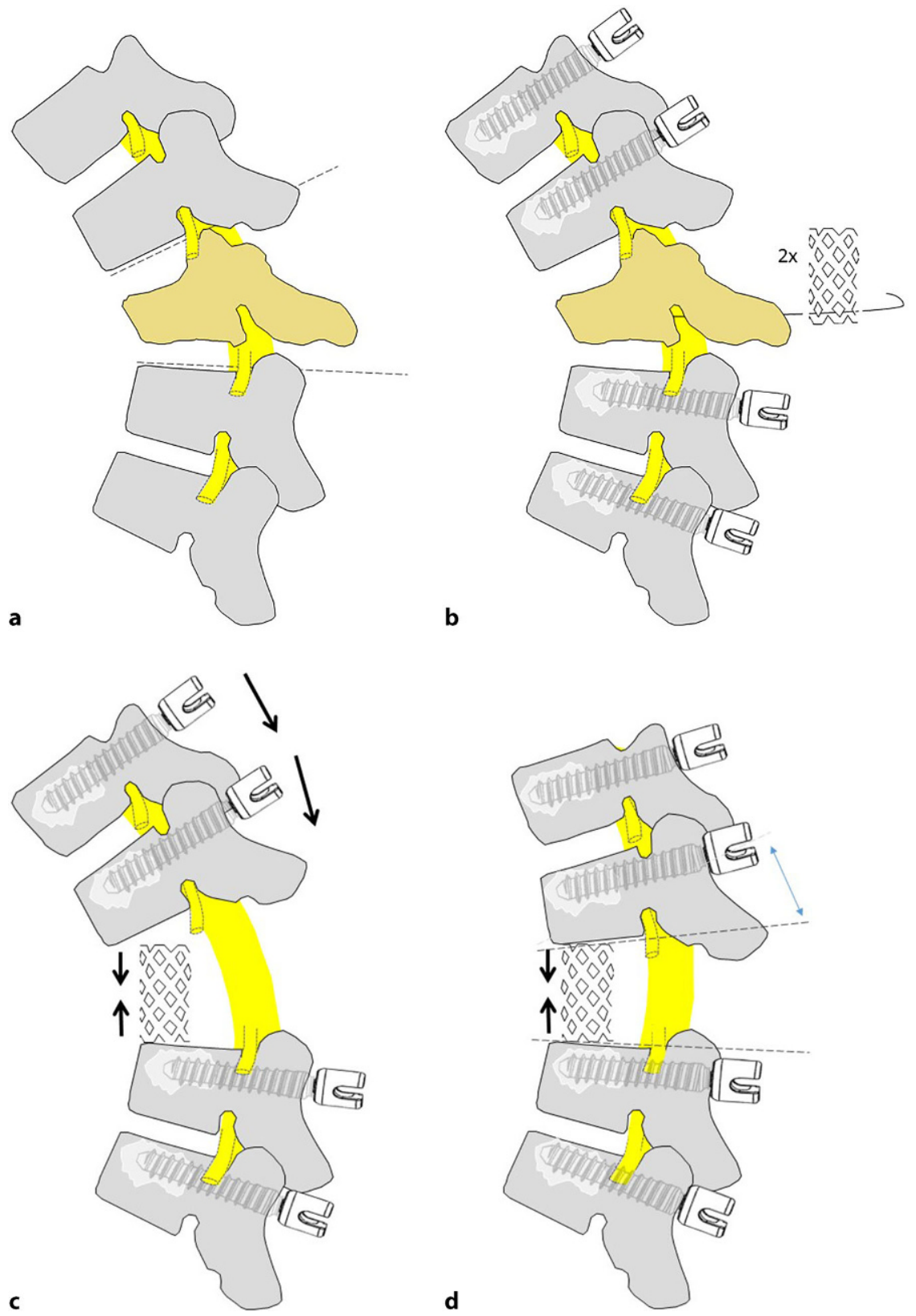

d

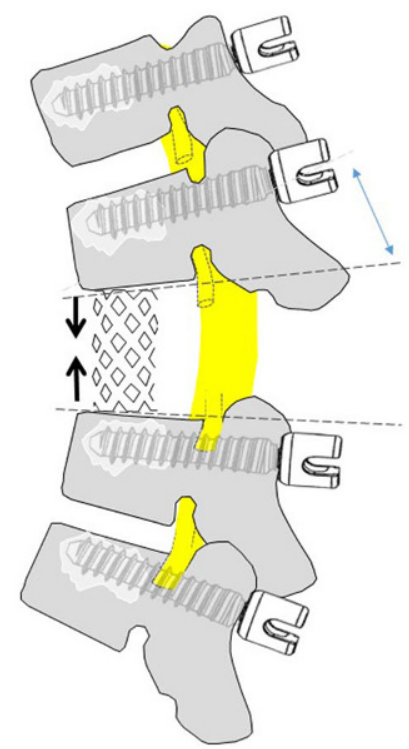

Abb. 1 ॥ Schematische Darstellung der "posterior vertebral column resection“.a Darstellung derFehlstellung. b Besetzen der kranialen und kaudalen Wirbel mit Pedikelschrauben und Definition der Resektionshöhen. c Einbringen des Mesh-Cages, der bei der späteren Kompression als Hypomochlion zur Deformitätenkorrektur wirkt. Über die Längsträger wird das Segment um bis zu zwei Drittel verkürzt. d Einbringen der Mesh-Cages und dorsale Kompression zur Reposition der Fehlstellung. Vermeidung der Kompression über kaudale Schrauben. (Mod. nach [4, 5])

Ausreißen von Schrauben oder CageSinterung bei schlechter Knochenqualität

\section{Operationsvorbereitungen}

- Röntgenbilder der Brustwirbelsäule in 2 Ebenen im Stehen

- Ganzwirbelsäulenaufnahme in 2 Ebenen im Stehen
- CT der BWS mit Bestimmung der Knochenqualität (qCT)

- MRT der BWS

- Bei fehlender qCT-Möglichkeit präoperative Durchführung einer Knochendichtemessung mittels Dual-Röntgen-Absorptiometrie (DXA)

- Blutverluste von über $1000 \mathrm{ml}$ sind bei dieser Operationsmethode möglich.
Diesem Blutverlust muss präoperativ durch eine ausreichende Bereitstellung von Erythrozytenkonzentraten Rechnung getragen werden

- Cell Saver (Cave: eingeschränkte Anwendung bei Tumoren bzw. Kontraindikation bei Spondylodiszitis)

\section{Instrumentarium}

- Dorsales Schrauben-Stab-System

- Mesh-Cage für ventrale Abstützung

- Wirbelsäulenstanzen und -rongeure

- Monopolare Messer- oder Spatelelektrode

- Bipolare Pinzetten

- Operationsmikroskop

- Highspeed-Fräse

- Gegebenenfalls synthetisches Knochenersatzmaterial zum Füllen des Cages im Gemisch mit reseziertem Knochen bzw. zur dorsalen Spondylodese

- Gegebenenfalls Anwendung eines Neuromonitorings

- Gegebenenfalls Anwendung eines Navigationssystems zur Schraubenpositionierung

\section{Anästhesie und Lagerung}

- Allgemeinnarkose

- Bauchlage auf Kissen, ggf. Concordlagerung mit Mayfield-Klemme

- Bei sehr ausgeprägten Kyphosen kann über ein Entknicken des Operationstisches nach Resektion des Wirbels die Reposition unterstützt werden

- Perioperative Antibiotikaprophylaxe

- Gabe von Tranexamsäure $1 \mathrm{~h}$ vor Schnitt 
Oper Orthop Traumatol 2019·31:311-320 https://doi.org/10.1007/s00064-019-0616-6

(c) Der/die Autor(en) 2019

\section{S. Sehmisch $\cdot$ W. Lehmann $\cdot$ M. Dreimann $\cdot$ S. Oberthür $\cdot$ L. Viezens $\cdot$ L. Weiser}

\section{Posterior vertebral column resection (PVCR) zur Korrektur von Hyperkyphosen bei osteoporotischen Frakturen der Brustwirbelsäule}

\section{Zusammenfassung}

Operationsziel. Die „posterior vertebral column resection" (PVCR) ist eine Reservetechnik zur Behandlung schwerer thorakaler Kyphosen mit bereits eingetretenen oder zeitnah drohenden neurologischen Komplikationen. Diese Technik sollte aufgrund des hohen Komplikationsspektrums nur ausgewählten Patienten empfohlen werden. Das Ziel der Operation ist die einzeitige Resektion des Wirbelkörpers über eine Kostotransversektomie mit einer $360^{\circ}$-Spondylodese. Nach Resektion des Wirbelkörpers gelingt eine Aufrichtung einer zuvor bestehenden kyphotischen Fehlstellung.

Indikationen. Ausgeprägte Kyphosen der Brustwirbelsäule (BWS) nach osteoporotischen Sinterungsfrakturen mit bereits eingetretenen oder zeitnah drohenden neurologischen Komplikationen auf einer oder mehreren Höhen.

Kontraindikationen. Allgemeine Kontraindikationen gegen chirurgische Eingriffe, ASA $>3$ (American Society of Anesthesiologists). Operationstechnik. Zuerst erfolgt eine Stabilisierung der Wirbelsäule mit mindestens 2 Wirbelkörpern kranial und kaudal der „vertebral column resection“ (VCR). Einzeitig werden anschließend eine Laminektomie mit Kostotransversektomie sowie die Resektion des Wirbelkörpers durchgeführt. Die ventrale Abstützung erfolgt durch das Einbringen eines Mesh-Cages in den ventralen Defekt. Durch die Kompression über die Längsträger wird die Fehlstellung reponiert und die MeshCages verklemmt. Abschließend wird die $360^{\circ}$ Spondylodese von dorsal durch Aufmeißeln der Wirbelgelenke und die Anlagerung von Knochen bzw. Knochenersatzmaterial komplettiert.

Weiterbehandlung. Funktionelle Nachbehandlung mit Vermeidung von Spitzenbelastungen und Behandlung der Osteoporose.

Ergebnisse. In einer von den Autoren durchgeführten retrospektiven Studie wurden 10 Patienten mit dieser Operationstechnik analysiert. Die Ergebnisse zeigen eine sehr gute Korrektur der kyphotischen Fehlstellung bei einem moderaten Komplikationsspektrum.

Schlüsselwörter

Wirbelsäule · Resektion · Wirbelkörper · Osteoporose $\cdot$ Spondylodese

\section{Posterior vertebral column resection for correction of kyphotic deformity due to osteoporotic fractures of the thoracic spine}

\section{Abstract}

Objective. The posterior vertebral column resection (PVCR) comprises a one-step resection of the vertebral body by a costotransversectomy together with a $360^{\circ}$ spondylodesis. After removing the vertebral body, straightening of the existing kyphotic malposition is possible.

Indications. Pronounced thoracic kyphosis after osteoporotic sintering fractures in one or more vertebral bodies.

Contraindications. General contraindications for surgical procedures, ASA >3 (American Society of Anesthesiologists).
Surgical technique. First, dorsal stabilization of the vertebral column on at least two levels cranial and caudal of the VCR. Next, in a one-step procedure the laminectomy with costotransversectomy and the resection of the vertebral body is done. The ventral defect gap is filled by a mesh cage to provide ventral support. By compression the malposition is reduced and the mesh cage is fixed into position. Finally the vertebrae joints are opened up using a chisel and bone or bone substitute is placed to complete the $360^{\circ}$ spondylodesis.
Postoperative management. Functional treatment without peak load exercises as well as appropriate osteoporosis treatment. Results. In a retrospective study 10 patients treated with this surgical technique were investigated. The results show a very good correction of the kyphotic maldeformity while the complications remain moderate.

Keywords

Kyphosis · Spine - Resection - Osteoporosis . Spinal fusion 


\section{Operationstechnik}

(•Abb. 2, 3, 4, 5, 6, 7, 8, 9, 10)

\section{Zugang und Setzen der Pedikelschraube}

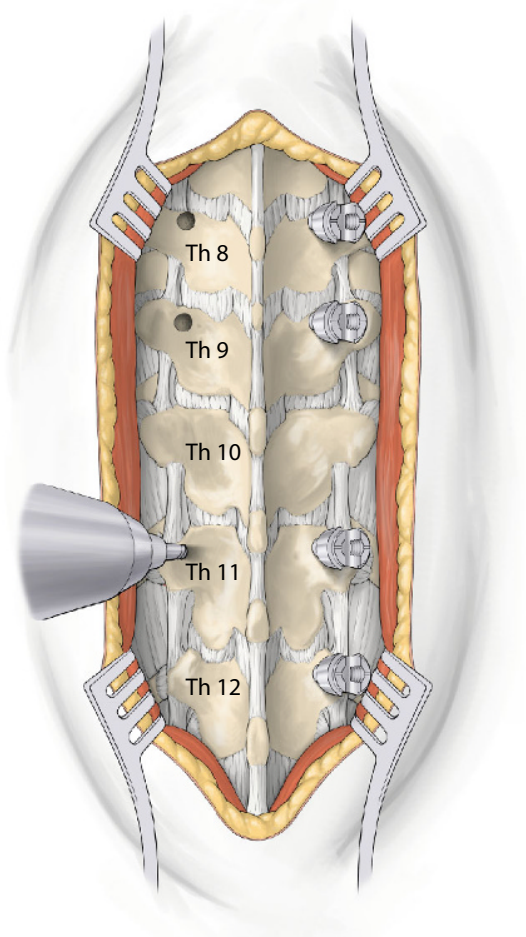

Abb. 2 \ Der Hautschnitt erfolgt über den Dornfortsätzen der betreffenden Segmente bzw. den Segmenten, die in die Spondylodese mit einbezogen werden. Nach Durchführung des Hautschnitts wird die weitere Präparation mit dem monopolaren Messer fortgesetzt. Zunächst werden die Dornfortsätze dargestellt. Anschließend Darstellung der Laminae. Dies gelingt mit dem Cobb-Rasparatorium und dem monopolaren Messer. Auf Höhe der geplanten "vertebral column resection" (VCR) muss jeweils bis zu den Rippenköpfchen präpariert werden. Die Blutstillung wird durch Abstopfen mit Kompressen erreicht. Währenddessen wird die andere Seite präpariert. Nach Darstellung der Laminae werden die Pedikelschrauben gesetzt. Auf Höhe der Brustwirbelsäule empfehlen die Autoren mindestens das Besetzen von 2 Wirbeln über und unter der geplanten VCR. Je nach Knochenqualität sollten fenestrierte Schrauben zur Zementaugmentation verwendet werden. Diese Operationsmethode erfordert eine hohe Primärstabilität, somit können längerstreckige Spondylodesen ( $>2$ Wirbel kranial und kaudal der PVCR) in Abhängigkeit von der Knochenqualität sinnvoll sein. Das Pedikulieren kann anhand anatomischer Landmarken, unter Röntgenkontrolle oder mit Hilfe eines Navigationssystems erfolgen. Nach Setzen der Schrauben muss eine Röntgenkontrolle in beiden Standardebenen durchgeführt werden

\section{Dekompression}

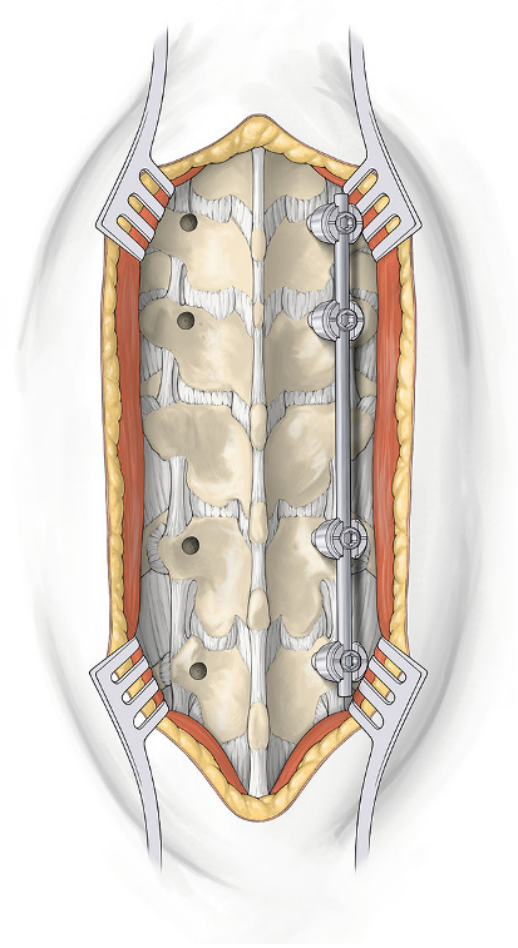

Abb. 3 ॥ Nach dem Setzen der Pedikelschrauben wird derSpinalkanal dekomprimiert. Es wird zunächst monolateral ein Längsträger montiert 


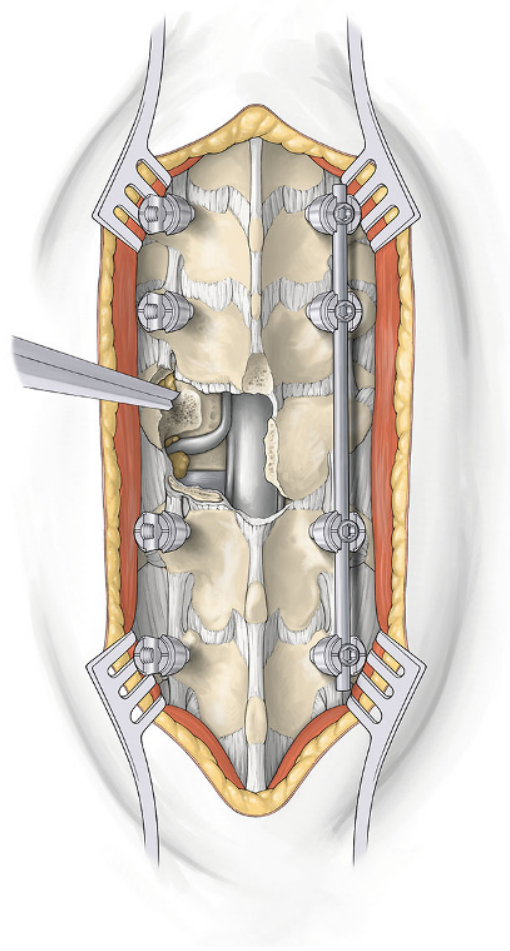

Resektion des Wirbelkörpers

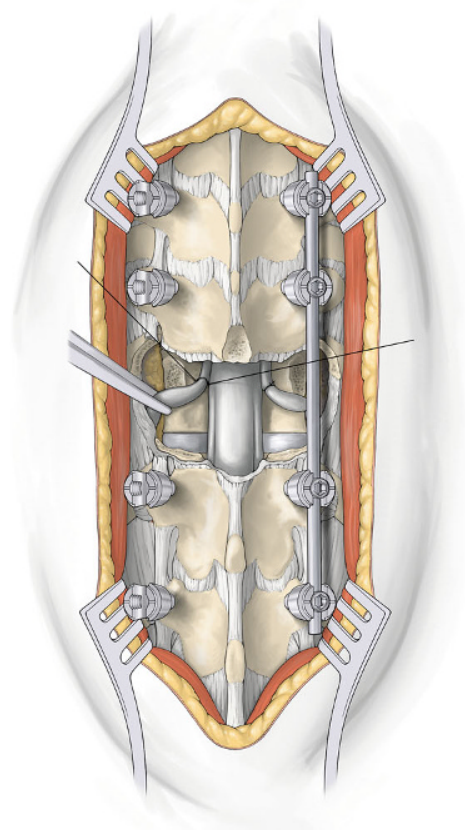

a
Abb. $5<$ Die Dekompression wird bis weit nach lateral mit Resektion des Rippenköpfchens fortgesetzt. Mit der Highspeed-Fräse wird die Lamina bzw. der Knochen jeweils ausgedünnt, um sie leichter mit der Stanze resezieren zu können. Nach Darstellung der Nervenwurzel wird der Längsträger umgebaut und die kontralaterale Seite analog präpariert
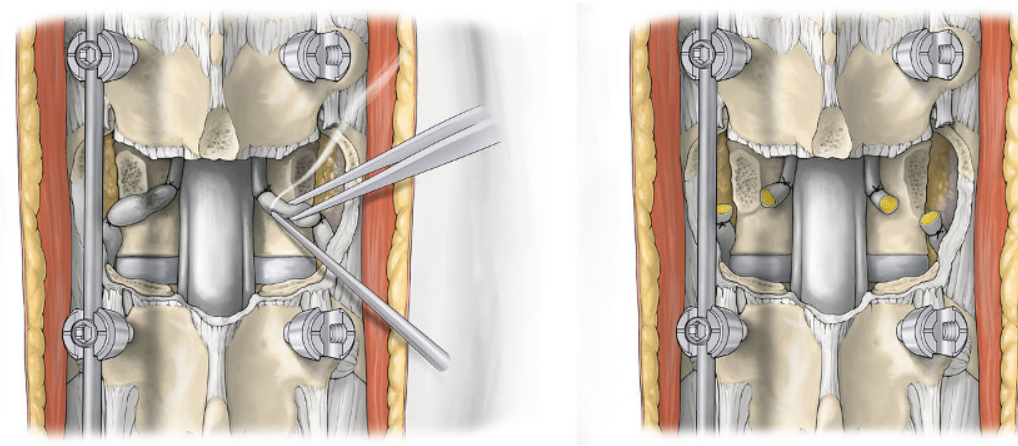

b c

Abb. 6 ॥ Nach Darstellung und Präparation der Nervenwurzel wird diese sowohl nach zentral als auch nach lateral mit einem 2-0 Vicryl-Faden ligiert (a). Die Wurzel wird anschließend zwischen den Ligaturen mit der bipolaren Pinzette koaguliert und anschließend scharf durchtrennt (b). Dies erfolgt beidseitig (c). Um stärkere Blutungen zu vermeiden, werden die epiduralen Venen vor der Wirbelkörperhinterkante mit der bipolaren Pinzette koaguliert (Reduktion der Stromstärke auf $20 \mathrm{~W}$, um Verletzungen der Dura zu vermeiden). Im Pedikel des zu resezierenden Wirbels wird mit der Highspeed-Fräse in den Korpus gefräst. In der Nähe der Dura empfiehlt sich der Einsatz eines Ultraschallmessers. Mit diesem kann die Resektion des Knochens nahe der Dura sicherer durchgeführt werden. Die Pedikelwand wird anschließend mit der Stanze entfernt. Nun kann das kraniale Bandscheibenfach mit dem Dissektor getastet und eröffnet werden. Nicht immer kann das Bandscheibenfach direkt mit dem Dissektor getastet werden. Um Verletzungen der angrenzenden Grund- und Deckplatte zu vermeiden, empfiehlt sich bei Unsicherheit die fluoroskopische Kontrolle. Unter Röntgen können die Bandscheibenfächer mit dem Dissektor markiert und identifiziert werden 


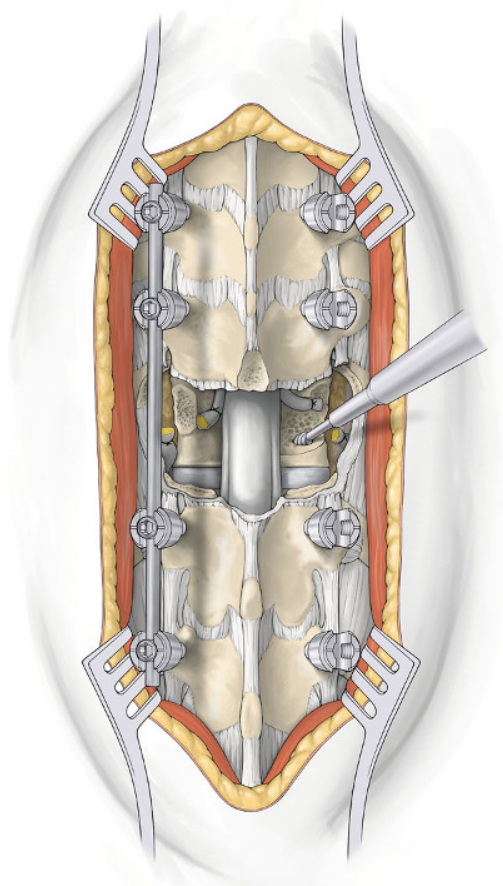

a

Abb. 7 A Mit der Highspeed-Fräse bzw. Stanzen wird der Wirbelkörper anschließend sukzessive entfernt (a). Die Grundplatte wird mit einer Kürette präpariert. Die kaudale Bandscheibe kann ebenfalls mit dem Dissektor pal piert und eröffnet werden (b). Anschließend wird auch der kaudale Wirbelkörper mit der Fräse und Stanzen sukzessive entfernt. Hierbei kann es zu stärkeren Blutungen kommen. Diese können mit synthetischen Hämostyptika gemildert werden. Während des gesamten Resezierens muss der Duraschlauch geschützt werden. An der thorakalen Wirbelsäule kann der Duraschlauch nur sehr wenig mobilisiert werden, da das Myelon auf diesen Höhen sehr empfindlich auf Druck reagiert. Aus diesem Grund erfolgt die weitere Präparation unter Vermeidung einer Kompression und segmentalen Distraktion der Dura. Nach Umsetzen des Längsträgers wird analog auf der kontralateralen Seite vorgegangen. Nach ausreichender Präparation und Resektion des Wirbels sind kranial die Grundplatte und kaudal die Deckplatte frei tastbar. Diese sollten bei der Präparation nicht verletzt werden. Nur so kann der Cage später als Hypomochlion zur Kyphosekorrektur verwendet werden. Zudem ist der gesamte Knochen dorsal des Myelons entfernt worden. Ebenso sollte sichergestellt sein, dass die gesamte Wirbelkörperhinterkante reseziert worden ist, da es andernfalls im Rahmen der Reposition/Kompression zur einem Vorwölben der verbliebenen Hinterkante in den Spinalkanal kommen könnte; ggf. ist hier ein L-förmiger Stößel sehr hilfreich. Die laterale und ventrale Kortikalis des Wirbels müssen bei Osteoporose-assoziierten Veränderungen nicht reseziert werden 


\section{Positionierung der Cages}

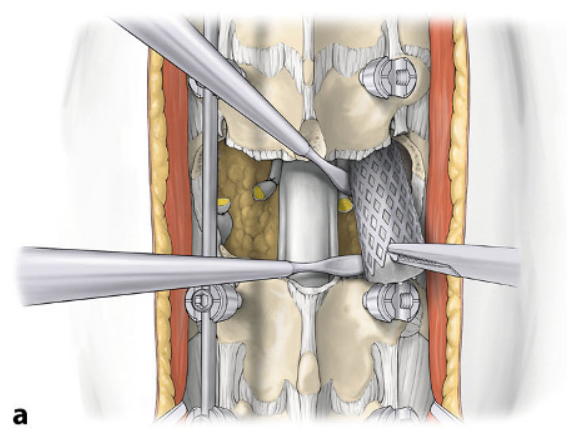

b

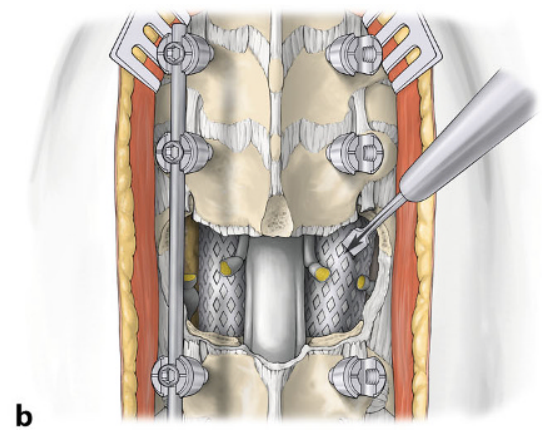

Abb. 8 \& Zur ventralen Abstützung empfehlen die Autoren die Verwendung von Mesh-Cages. Die Cages sind in verschiedenen Durchmessern verfügbar und können stufenlos an die Länge des Defektes angepasst werden. Mit einem Schleppmesser kann die Cage-Länge ermittelt werden. Die Autoren empfehlen entweder das Einbringen eines großkalibrigen zentralen Cages oder zweier kleiner in paralleler Ausrichtung. Letztere Technik wird hier favorisiert, da durch die kleinere Cage-Größe eine geringere Gefährdung für die Dura besteht. Vor dem Einbringen werden die Cages mit Knochen oder Knochenersatzmaterial gefüllt. Unter strengster Vermeidung eines Durakontaktes werden die Cages an der Dura vorbei eingebracht und in kraniokaudaler Richtung mit Hilfe von Stößeln positioniert (a). Bei Verwendung von 2 Cages wird nach Einbringen des ersten der Längsträger umgebaut und kontralateral der zweite Cage eingebracht. Unter Röntgenkontrolle erfolgt die definitive Cage-Positionierung (b). Prinzipiell empfehlen die Autoren eine zentrale bis ventrale Positionierung. Zur Blutungskontrolle erfolgt nach einer gründlichen Wundspülung die Anlagerung von Hämostyptikaschwämmen
Dorsale Stabilisierung

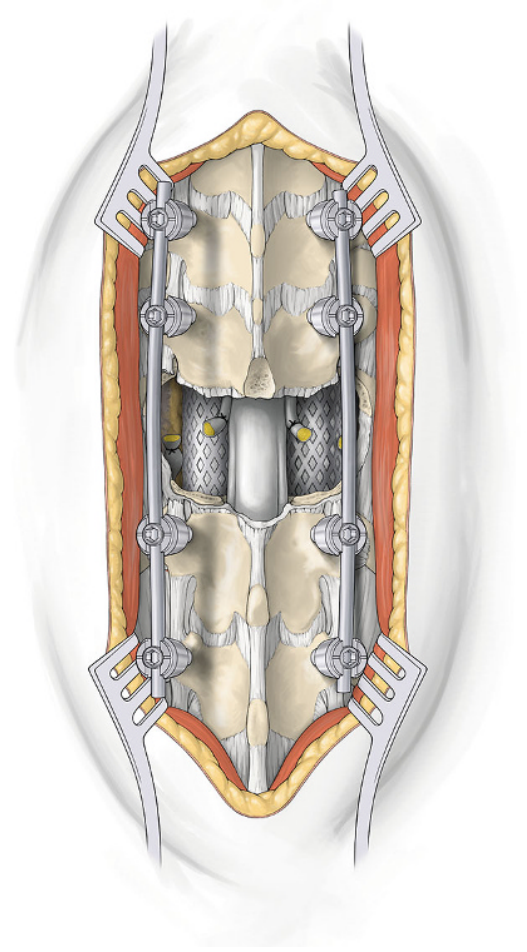

Abb. 9 \& Nach der Positionierung der Cages werden die Längsträger nach anatomischem Vorbiegen eingebracht. Über die Längsträger und die Aufrichtung des in Kyphose positionierten OP-Tisches werden die Reposition der thorakalen Wirbelsäule erreicht und die Cages unter Kompression gebracht. Die Reposition wird in mehreren Teilschritten und wiederholt wechselseitig durchgeführt. Hierbei ist darauf zu achten, dass die Kompression um ein Drittel der Wirbelkörperhöhe als unkritisch, zwei Drittel als kritisch und drei Drittel als gefährlich für postoperative neurologische Defizite angesehen wird (• Abb. 1) 


\section{Dorsale Spondylodese und Wundverschluss}

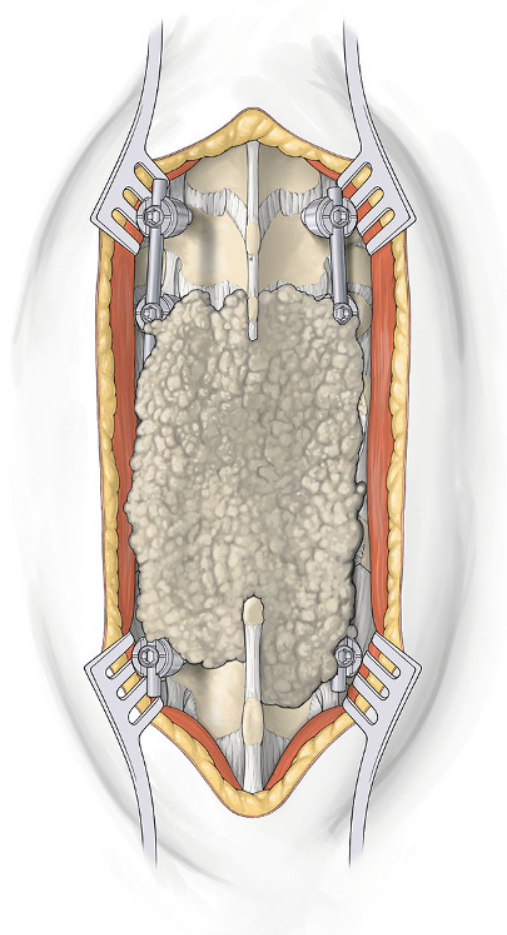

Abb. $10 \Delta$ Nach definitiver Stabilisierung wird die Dura mit einem Hämostypikum abgedeckt. Mit der großen Luer-Zange oder der Fräse werden die kranialen und kaudalen Laminae bis auf den spongiösen Knochen angefrischt. Die in der Instrumentation eingefassten Facettengelenke werden mit dem Meißel eröffnet. Der Situs wird nun erneut gründlich gespült. Anschließend werden die Laminae und der Defekt der Dekompression mit Knochen, einem synthetischen Knochenersatz oder einem Gemisch aus beidem bedeckt. Es erfolgt die Einlage von zwei 12-Ch-Redondrainagen. Die Faszie wird zunächst in Einzelknopftechnik adaptiert und anschließend durch eine fortlaufende Naht wasserdicht verschlossen. Darüber wird die Subkutanschicht und abschließend die Haut genäht. Die Drainagen werden erst nach Umlagerung ins Bett geöffnet

\section{Postoperative Behandlung}

- Überwachung auf einer IMC/ Intensivstation für $24 \mathrm{~h}$

- Direkt postoperative neurologische Überwachung und regelmäßige Kontrolle

- Mobilisation ab dem 1. postoperativen Tag

- Funktionelle Nachbehandlung unter Vermeidung von Spitzenbelastungen

- Regelmäßige Wundkontrollen. Behandlung der Osteoporose ggf. Optimierung einer vorbestehenden Therapie

\section{Fehler, Gefahren, Komplikationen}

- Unbedingter schonender Umgang mit dem Rückenmark - insbesondere Vermeidung einer Distraktion

- Vermeidung der intraoperativen Instabilität durch wechselseitiges Einbringen eines unilateralen Längsträgers

- Durchführung der PVCR über maximal 3 Segmente

\section{Fallbeispiel}

Eine 72-jährige Patientin wies eine glukokortikoidinduzierte Osteoporose (GIOP) bei Myasthenia gravis auf. Die Patientin berichtete über ausgeprägte Schmerzen (visuelle Analogskala [VAS] 8) und eine zunehmende Dyspnoe. Die Magnetresonanztomographie (MRT) zeigt eine thorakale Myelopathie (• Abb. 11). In der Diagnostik wird eine thorakale Kyphose bei Fraktur von T11 und 9 mit einem Cobb-Winkel von $79^{\circ}$ festgestellt.

Aufgrund einer drohenden neurologischen Verschlechterung wird die Indikation zur PVCR gestellt. Nach Durchführung der PVCR zeigt sich eine deutliche Stellungsverbesserung mit einem postoperativen Cobb-Winkel von $31^{\circ}$ (Korrektur von $48^{\circ}$; • Abb. 12).

In $\bullet$ Abb. 13 sind die Ergebnisse der radiologischen Kontrolle nach 9 Monaten dargestellt. Klinisch beschreibt die Patientin eine deutliche Beschwerdelinderung mit Reduktion der Schmerzen (VAS 2-3) und deutlicher Verbesserung der Dyspnoe.

\section{Ergebnisse}

Die PVCR ist eine selten angewendete Operationstechnik und sollte nur bei speziellen Indikationen Verwendung finden. Primäre Therapieverfahren sind die konservative Behandlung von Osteoporoseassoziierten Frakturen sowie die Kyphoplastie mit und ohne posteriore Stabilisierung.

In einer von Mitautoren dieses Artikels verfassten retrospektiven Studie [4] wurden 10 Patienten analysiert, die mit dieser Operationstechnik von 2012 bis 2015 stabilisiert wurden. Das Durchschnittsalter der untersuchten $\mathrm{Pa}$ tienten betrug 73,3 Jahre (58-85 Jahre). Mit Ausnahme einer Höhe wurden alle Pedikelschrauben mit einer additiven Zementaugmentation eingebracht. Die durchschnittliche Operationszeit betrug $318 \pm 62 \mathrm{~min}$, und der durchschnittliche Blutverlust betrug $1540 \pm 745 \mathrm{ml}$. Der Nachbeobachtungszeitraum der Patienten lag bei durchschnittlich 14 Monaten (3-36 Monate).

Der präoperative Rückenschmerz wurde auf der visuellen Analogskala (VAS) mit 8,6 $\pm 2,0$ (VAS: 4-10) beschrieben. Bei der letzten Verlaufskontrolle zeigte sich ein deutlicher Rückgang auf 5,0 $\pm 1,4$ (VAS: $3-8$ ).

Die Analyse des Oswestry Disability Index (ODI) zeigte präoperativ bei 2 Patienten eine starke Einschränkung (41-60\%), bei 5 eine sehr starke Funktionsminderung (61-80\%) und bei $3 \mathrm{~Pa}$ tienten eine komplette Funktionseinschränkung (81-100\%). Durch die PVCR berichteten 6 Patienten über eine starke Einschränkung (41-60\%), 3 über eine sehr starke und weiterhin 1 Patient über eine komplette Funktionsminderung.

Der Frankel-Score verbesserte sich bei 3 Patienten von D auf E oder zeigte keine Veränderung von prä- zu postoperativ.

Die radiologische Nachuntersuchung zeigte eine Verbesserung der kyphotischen Fehlstellung von $20^{\circ} \pm 10^{\circ}$. Präoperativ wurde eine Kyphose von durchschnittlich $25^{\circ} \pm 14^{\circ}\left(5-53^{\circ}\right)$ gemessen. Zum letzten Nachuntersuchungszeitpunkt betrug der Cobb-Winkel $8^{\circ} \pm 7^{\circ}$ $\left(5^{\circ}-21^{\circ}\right)$. Im Vergleich $\mathrm{zu}$ angrenzenden gesunden Segmenten wurde das 

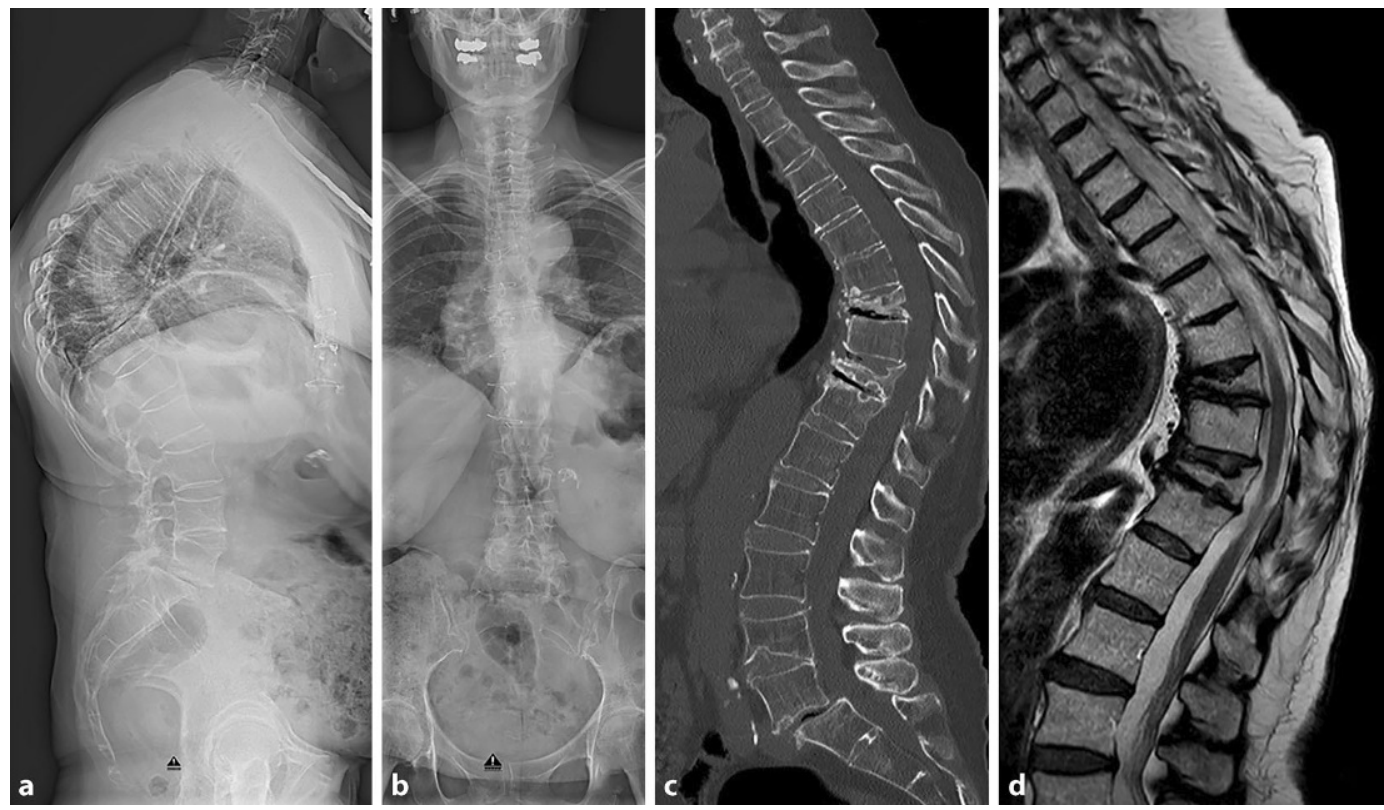

Abb. $11<$ Fallbeispiel. a Sagittale Ansicht der Ganzwirbelsäulenaufnahme. b Koronare Aufnahme der Ganzwirbelsäulenaufnahme. c Sagittale CT-Ansicht mit Darstellung der stattgehabten Frakturen. d MRT mit Demonstration keiner hochgradigen neuronalen Bedrängung
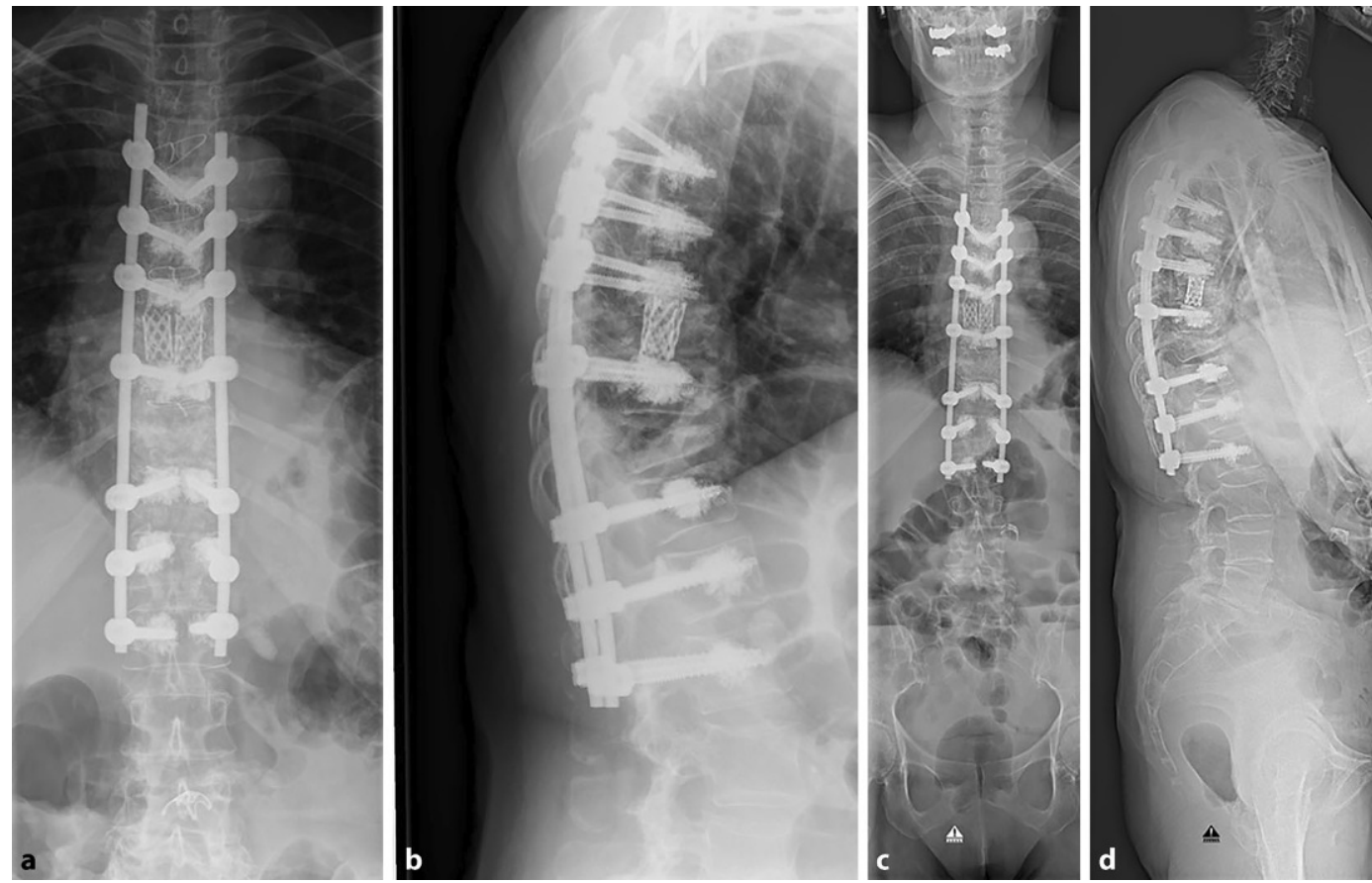

Abb. $12 \triangleleft$ Postoperative Kontrolle nach "posterior vertebral column resection" (PVCR). a Brustwirbelsäule (BWS) a.-p. b BWS seitlich. c Ganzwirbelsäule a.-p.d Ganzwirbelsäule seitlich

betroffene Segment um 23\% (0-36\%) verkürzt. Eine knöcherne Fusion wurde bei den Patienten innerhalb von 6 Monaten erzielt.

Bei 3 Patienten wurden im Beobachtungszeitraum Komplikationen beob- achtet. In einem Fall kam es $\mathrm{zu}$ einer intraoperativen mechanischen Komplikation, sodass die Instrumentationsstrecke verlängert wurde. In einem anderen Fall trat eine revisionspflichtige chirurgische Wundinfektion und in einem weiteren Fall eine deutliche klinisch/ neurologische Verschlechterung nach initialer Besserung auf. Eine Implantatfehllage konnte dabei ausgeschlossen werden. 


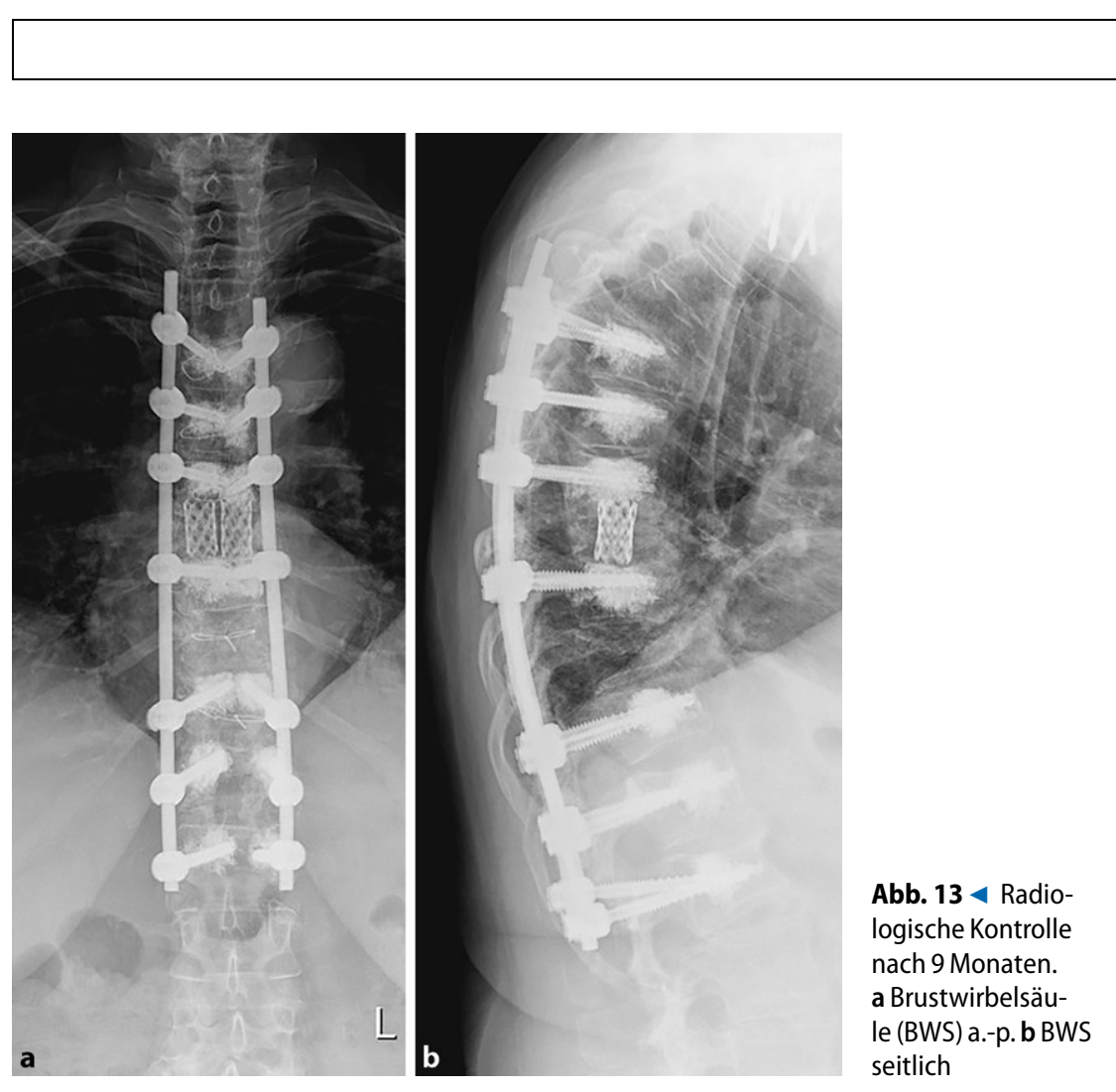

Lesetipp

\section{Bildgebende Diagnostik des Fußes}

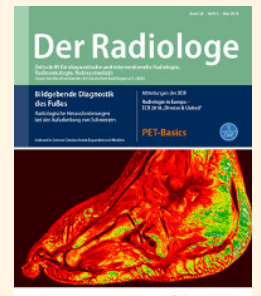



"Der menschliche Fuß ist ein Kunstwerk aus 26 Knochen, 107 Bändern und 19 Muskeln." Dieses Zitat von Leonardo da Vinci zeigt, dass es lohnenswert ist, sich mit den wesentlichen Aspekten der Anatomie, Pathologie und den Bildgebungsbefunden, die dem Radiologen bei der diagnostischen Aufarbeitung von Schmerzen am Fuß begegnen, zu beschäftigen.

Informieren Sie sich in der Ausgabe 05/2018 von Der Radiologe unter anderem über

- Erwartungen des Orthopäden vom Radiologen bei der bildgebenden Diagnostik

- Typische Frakturen - welche sollte der Radiologe kennen?

- Knorpelbildgebung, Entzündungen und Fehlbildungen

- Tumoren und tumorähnliche Läsionen im Fuß

2. Zeng Y, Chen Z, Guo Z, Qi Q, Li W, Sun C (2014) The posterior surgical treatment for focal kyphosis in upper-middle thoracic spine. Eur Spine J 23(11):2291-2298

3. Lee $B H$, Hyun $S J$, Han $S$, Kim KJ, Jahng $T A$, Kim YJ, Kim HJ (2018) Surgical and radiological outcomes after posterior vertebral column resection according to the surgeon's experience. Medicine (Baltimore) 97(34):e11660

4. Dreimann $M$, Hempfing $A$, Stangenberg $M$, Viezens L, Weiser L, Czorlich P, Eicker SO (2018) Posterior vertebral column resection with 360 degree osteosynthesis in osteoporotic kyphotic deformity and spinal cord compression. Neurosurg $\operatorname{Rev} 41(1): 221-228$

5. Dreimann M, Hoffmann M, Viezens L et al (2017) Reducing kyphotic deformity by posterior vertebral column resection with $360^{\circ}$ osteosynthesis in metastatic epidural spinal cord compression (MESCC). Eur Spine J 26:113-121 